\title{
Triaging of Culture Conditions for the Enhanced Discovery of Secondary Metabolites from Different Bacteria
}

\author{
Jenny Schwarz ${ }^{1}$ and Stephan Lütz ${ }^{1, *}$ \\ 1 Chair of Bioprocess Engineering, Department of Chemical and Biochemical Engineering, TU Dortmund \\ University, Emil-Figge-Straße 66, 44227 Dortmund, Germany \\ * Correspondence: stephan.luetz@tu-dortmund.de
}

Received: date; Accepted: date; Published: date

\begin{abstract}
Over the past decade, the One Strain Many Compounds (OSMAC) approach has been established for silent gene cluster activation and elicitation of secondary metabolite production, but so far the full secondary metabolome of a biosynthetically promising bacterium has not been elucidated yet. Here, we investigate the ability of seven categories of OSMAC conditions to elicit new mass features from bacterial strains with little literature coverage but high biosynthetic potential. The strains B. amyloliquefaciens DSM7, C. coralloides DSM2259, P. fallax HKI727, R. jostii DSM44719 and S. griseochromogenes DSM40499 were selected after genome mining with antiSMASH. After cultivation under OSMAC conditions, the generated extracts were subjected to $\mathrm{LC} / \mathrm{MS}$ and MZmine analysis to determine new mass features, expressed gene clusters and evaluate the tested culture conditions. 4 predicted compounds, bacillibactin, desferrioxamine B, myxochelin A and surfactin, were identified and up to 147 new mass features were detected in the generated extracts, which greatly surpasses the number of predicted gene clusters. Among the new mass features are bioactive compounds which were so far unreported for the strains such as cyclo-(TyrPro) from DSM7 and nocardamin from DSM2259. Furthermore, the tested culture conditions were evaluated regarding their suitability for the generation of new mass features from the selected strains and promising new starting points for further screenings are postulated. Especially culture conditions with little prior literature coverage are responsible for the activation of secondary metabolite production.
\end{abstract}

Keywords: mass spectrometry; OSMAC approach; natural products; silent BGC activation; bioinformatics; screening

\section{Introduction}

Secondary metabolites, of which currently about 300,000 are known, are diverse and often complex natural products occurring in all living beings. The genes coding for secondary metabolite producing enzymes are often clustered with their expression regulated by parameters including growth phase and cell differentiation [1,2]. Due to their diverse biological profile, e.g. antibiotic, cytostatic or other activities, they are of high interest for pharmaceutical research $[2,3]$.

The traditional approach of natural product discovery from microorganisms comprises bioactivity-guided screenings of microbial extract libraries obtained from existing strain collections $[4,5]$ or newly isolated microorganisms [6,7]. Especially actinomycetes are regarded as biosynthetically promising sources [4]. With the development of new sequencing technologies, the number of freely available fully sequenced genomes in public databases, e.g. National Center for Biotechnology Information (NCBI), increased and held more than 200,000 prokaryotic genome sequences as of 2020 with different assembly levels. This made predictions regarding potentially producible secondary metabolites possible, e.g. by bioinformatics analyses of genomes using tools 
like antiSMASH [8]. These analyses allow a new approach for secondary metabolite discovery: the discovery of previously unidentified natural products from the broad spectrum of the sequenced genomes of isolated and deposited strains [9]. As not all biosynthetic gene clusters (BGCs) for secondary metabolites present on a genome are expressed under a certain set of fermentation conditions [10], the activation of these silent BGCs can be achieved by genetic modifications, e.g. ribosome engineering, the manipulation of regulators or quorum sensing systems, and heterologous expression [11,12]. Heterologous expression can also be used to confirm an assumed encoding function of a BGC as was shown by the example of pacidamycin biosynthesis [13]. Besides genetic modification, activation of a broad spectrum of BGCs can be achieved by co-cultivation [4] or the One Strain, Many Compounds approach (OSMAC), which focuses on the production of many compounds by one microbial source [14].

The OSMAC approach comprises the alteration of easily adaptable cultivation conditions, e.g. media composition, $\mathrm{pH}$, temperature, addition of enzyme inhibitors, oxygen supply, or culture vessel [14]. Error! Reference source not found. shows examples of pharmaceutically active new compounds obtained from successful OSMAC experiments using easily applicable changes of cultivation conditions.

Table 1. Examples of activation of silent BGCs by altering cultivation conditions according to the OSMAC approach.

\begin{tabular}{ccccc}
\hline Strain & Natural product & Altered conditions & Properties & Reference \\
\hline $\begin{array}{c}\text { Sphaeropsidales } \\
\text { sp. F-24'707 }\end{array}$ & 42 cladospirones & variation of media & antibacterial and antifungal & {$[15]$} \\
\hline $\begin{array}{c}\text { Streptomyces } \\
\text { sp. MBT27 }\end{array}$ & $\begin{array}{c}\text { quinazolinone A \& } \\
\text { B }\end{array}$ & $\begin{array}{c}\text { variation of carbon } \\
\text { sources }\end{array}$ & $\begin{array}{c}\text { anti-inflammatory, antitumor, } \\
\text { antimicrobial and anti-fungal } \\
\text { properties }\end{array}$ & {$[16]$} \\
\hline $\begin{array}{c}\text { Aspergillus sp. } \\
\text { LS34 }\end{array}$ & 9 new compounds & $\begin{array}{c}\text { solid rice medium \& } \\
\text { potato dextrose } \\
\text { medium }\end{array}$ & $\begin{array}{c}\text { cytotoxic activity against cancer } \\
\text { cell lines/ active against } \\
\text { pathogenic S. aureus }\end{array}$ & {$[17]$} \\
\hline
\end{tabular}

Additives such as solvents, heavy metals, tensides, precursors, and other small molecule elicitors are also useful for the elicitation of secondary metabolite production in microorganisms. Chen reports that organic solvents can either increase (DMSO, EtOH) or decrease (THF, DMF) the production of antibiotics such as tetracenomycin and thiostreptone in Streptomyces strains [18]. A proposed mechanism of action is the induction of heat shock response through ethanol [19].

Weinberg identified three heavy metals, $\mathrm{Mn}^{2+}, \mathrm{Fe}^{3+}$, and $\mathrm{Zn}^{2+}$, as key trace metals in secondary metabolism [20]. The same trend can be seen in Paranagama's study on the influence of the addition of heavy metal ions and heat shock on fungal secondary metabolite production profiles: the plantassociated fungus Paraphaeosphaeria quadriseptata produced more monocillin I when supplemented with $0.5 \mathrm{mM} \mathrm{ZnSO}_{4}$ solution [21]. Heavy metals and solvents as abiotic additives constitute an interesting addition to the OSMAC approach.

A further promising approach for secondary metabolite induction is co-cultivation which takes advantage of inter-species communication. The term co-cultivation designates growth of two or more microorganisms at once, which facilitates communication between the strains [22]. Cell-cell-contact and signal molecules can induce a change of the metabolic profile and often lead to the expression of previously silent BGCs $[11,23]$. It was shown that co-cultivation of various marine bacteria with terrestrial bacteria enhances the antibiotic production.

A literature survey has shown, that OSMAC studies have been conducted increasingly in the past 8 years and that some conditions are used more often than others. (Figure 1) 


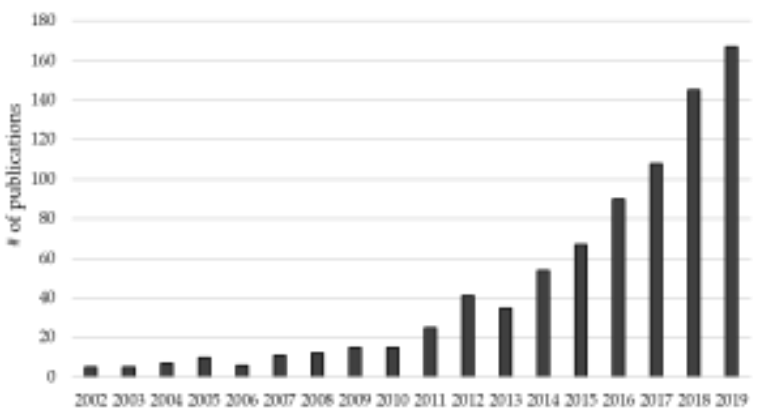

(a)

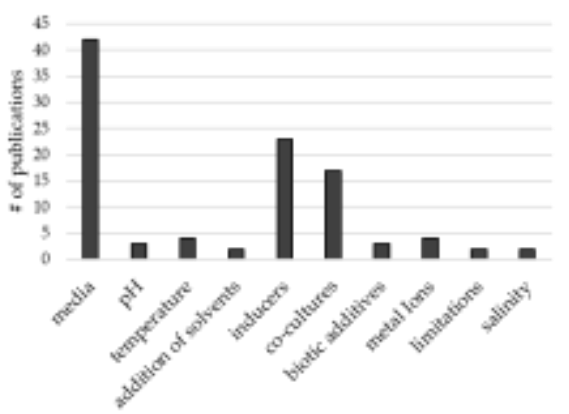

(b)

Figure 1. (a ) Number of publications listed on Google Scholar with the keywords "bacteria OSMAC" from 2002 onwards (including research papers, reviews, theses, book chapters, distribution see Figure S1); ( b ) Number of research papers and theses among those from ( a ) dealing with certain sets of culture conditions applied to bacterial cultures.

While the use of different media, addition of inducer molecules and use of co-culture for the increase of natural product titers and for the activation of silent BGCs in bacterial cultures are commonly found in literature, other options such as the addition of solvents or biotic additives, e.g. heat-killed cells, are not extensively covered.

In this study, we contribute to expanding the knowledge of the activation of bacterial secondary metabolite BGCs and the biosynthetic potential of selected bacteria. Five fully sequenced, but underinvestigated and biosynthetically promising bacterial strains were identified via genome mining. The activation of their BGCs was investigated by using a broad range of culture conditions: biotic additives (addition of supernatant or autoclaved cell pellet of an inducer microbe) and abiotic additives, i.e. solvents, as well as complex media, minimal media, $\mathrm{Fe}^{3+}-$ and $\mathrm{Mg}^{2+}-\mathrm{and}^{\mathrm{PO}_{4}{ }^{3-}}$ limitations, oxygen limitation and temperature changes. The tested culture conditions comprise well established as well as less frequently used conditions in secondary metabolite discovery and are evaluated regarding their suitability for the generation of new mass features from the strains. Based on their potential to increase and diversify secondary metabolite production, $\mathrm{PO}_{4}^{3--l i m i t e d ~} \mathrm{M} 9$ medium and $\mathrm{Fe}^{3+}$-limited GMS medium and several biotic additives are proposed as promising starting points for further screenings. Furthermore, it was noted that $70 \%$ of the new mass features are condition-specific, i.e. they were only detected under exactly one culture condition. This observation led us to the conclusion that especially broad screenings of carefully selected strains are promising for future secondary metabolite discovery.

\section{Materials and Methods}

Genome Mining The online-tool antiSMASH bacterial version 3.0 [8] was used to examine fully sequenced genomes of 42 bacteria from the phyla of actinomycetes, firmicutes and proteobacteria. The percentage of the genomes dedicated to secondary metabolite production, classes of the encoded secondary metabolites and the origin of the database BGCs were examined. A complete list of the examined bacterial strains and their NCBI accession numbers can be taken from the supplementary material (Table S1).

Organisms and Media The control groups of Bacillus amyloliquefaciens DSM7, Corallococcus coralloides DSM2259, Pyxidicoccus fallax HKI727, Rhodococcus jostii DSM44719, and Streptomyces griseochromogenes DSM40499 were cultivated according to recommendations by DSMZ (German Collection of Microorganisms and Cell Cultures). B. amyloliquefaciens DSM7 was cultivated on nutrient medium (5.0 g. $\mathrm{L}^{-1}$ peptone from meat, $3.0 \mathrm{~g} \cdot \mathrm{L}^{-1}$ meat extract, $\mathrm{pH} 7.0$ ), C. coralloides DSM2259 was grown on SP medium $\left(1.0 \mathrm{~g} \cdot \mathrm{L}^{-1}\right.$ raffinose, $1.0 \mathrm{~g} \cdot \mathrm{L}^{-1}$ sucrose, $1.0 \mathrm{~g} \cdot \mathrm{L}^{-1}$ galactose, $5.0 \mathrm{~g} \cdot \mathrm{L}^{-1} \mathrm{soluble}$ starch, $2.5 \mathrm{~g} \cdot \mathrm{L}^{-1}$ casitone, $0.5 \mathrm{~g} \cdot \mathrm{L}^{-1} \mathrm{MgSO}_{4} \times 7 \mathrm{H}_{2} \mathrm{O}, 0.25 \mathrm{~g} \cdot \mathrm{L}^{-1} \mathrm{~K}_{2} \mathrm{HPO}_{4}$, $\mathrm{pH} 7.4$ ), and P. fallax HKI727 on MD1 medium (3.0 g. $\mathrm{L}^{-1}$ casitone, $0.7 \mathrm{~g} \cdot \mathrm{L}^{-1} \mathrm{CaCl}_{2} \times 2 \mathrm{H}_{2} \mathrm{O}, 2.0 \mathrm{~g} \cdot \mathrm{L}^{-1} \mathrm{MgSO}_{4} \times 7 \mathrm{H}_{2} \mathrm{O}, 1 \mathrm{~mL}$ Trace Element Solution (TES) SL4, $0.5 \mathrm{mg} \cdot \mathrm{L}^{-1}$ vitamin $\mathrm{B}_{12}$, $\mathrm{pH} 7.0$ ) with the following composition of TES SL4: EDTA 
$\left(0.5 \mathrm{~g} \cdot \mathrm{L}^{-1}\right), \mathrm{FeSO}_{4} \times 7 \mathrm{H}_{2} \mathrm{O}\left(0.2 \mathrm{~g} \cdot \mathrm{L}^{-1}\right), \mathrm{ZnSO}_{4} \times 7 \mathrm{H}_{2} \mathrm{O}\left(0.01 \mathrm{~g} \cdot \mathrm{L}^{-1}\right), \mathrm{MnCl}_{2} \times 4 \mathrm{H}_{2} \mathrm{O}\left(0.003 \mathrm{~g} \cdot \mathrm{L}^{-1}\right), \mathrm{H}_{3} \mathrm{BO}_{3}$ $\left(0.03 \mathrm{~g} \cdot \mathrm{L}^{-1}\right), \mathrm{CoCl}_{2} \times 6 \mathrm{H}_{2} \mathrm{O}\left(0.02 \mathrm{~g} \cdot \mathrm{L}^{-1}\right), \mathrm{CuCl}_{2} \times 2 \mathrm{H}_{2} \mathrm{O}\left(0.001 \mathrm{~g} \cdot \mathrm{L}^{-1}\right), \mathrm{NiCl}_{2} \times 6 \mathrm{H}_{2} \mathrm{O}\left(0.002 \mathrm{~g} \cdot \mathrm{L}^{-1}\right), \mathrm{Na}_{2} \mathrm{MoO}_{4}$ $\times 2 \mathrm{H}_{2} \mathrm{O}\left(0.003 \mathrm{~g} \cdot \mathrm{L}^{-1}\right) . \quad$ R. jostii DSM44719 was cultivated on TSB medium $\left(17.0 \mathrm{~g} \cdot \mathrm{L}^{-1}\right.$ casitone, $3.0 \mathrm{~g} \cdot \mathrm{L}-$ ${ }^{1}$ soy peptone, $2.5 \mathrm{~g} \cdot \mathrm{L}^{-1} \mathrm{D}(+)$ glucose, $\left.5.0 \mathrm{~g} \cdot \mathrm{L}^{-1} \mathrm{NaCl}, 3.0 \mathrm{~g} \cdot \mathrm{L}^{-1} \mathrm{~K}_{2} \mathrm{HPO}_{4}, \mathrm{pH} 7.3\right)$ and cultures of $S$. griseochromogenes DSM40499 were grown on GYM medium $\left(4.0 \mathrm{~g} \cdot \mathrm{L}^{-1}\right.$ glucose, $4.0 \mathrm{~g} \cdot \mathrm{L}^{-1}$ yeast extract, $10.0 \mathrm{~g} \cdot \mathrm{L}^{-1}$ malt extract, $\mathrm{pH}$ 7.2). A detailed list of used chemicals and suppliers can be taken from Table S2.

Growth conditions Cryo-cultures of all strains were stored at $-20^{\circ} \mathrm{C}$ in $10 \%(\mathrm{v} / \mathrm{v})$ glycerol. Inocula were prepared by incubating $1 \mathrm{~mL}$ of the cryo-culture in $19 \mathrm{ml}$ of medium in a baffled $100 \mathrm{~mL}$-flask on an orbital shaker at $150 \mathrm{rpm}$ and $30^{\circ} \mathrm{C}\left(28^{\circ} \mathrm{C}\right.$ for $R$. jostii) for $15-72 \mathrm{~h}$ depending on the strain $(B$. amyloliquefaciens for $15 \mathrm{~h}, \mathrm{C}$. coralloides for $15 \mathrm{~h}, P$. fallax for $72 \mathrm{~h}, \mathrm{R}$. jostii for $43 \mathrm{~h}, \quad$ S. griseochromogenes for $15 \mathrm{~h}$ ). For the main culture, $10 \%$ inoculum was used in $90 \mathrm{ml}$ fresh medium in baffled $500 \mathrm{~mL}$ flasks and cultivated on an orbital shaker at $150 \mathrm{rpm}$ for the indicated period of time ( $B$. amyloliquefaciens and S. griseochromogenes for 3 days, C. coralloides, $P$. fallax and $R$. jostii for 7 days).

OSMAC experiments Variations of culture conditions comprised oxygen limitation (use of unbaffled flasks), temperature $\left(25^{\circ} \mathrm{C}, 35^{\circ} \mathrm{C}, 40^{\circ} \mathrm{C}\right)$, use of 3 different complex media per strain (SP medium, TSB medium, CY/H medium, MD1 medium, MD1+G medium, LB medium, GYM medium, NB medium, Landy medium, Luria medium), use of minimal media (M9 medium and GMS medium) as well as $\mathrm{Mg}^{2+}$, $\mathrm{PO}_{4}^{3--}$ or $\mathrm{Fe}^{2+-}$ limited versions thereof. (Tables S3-S8) Acetonitrile (ACN), dimethyl sulfoxide (DMSO), ethanol (EtOH) and toluene (Tol) were used as abiotic additives at $0.5 / 1 / 3 / 6$ Vol\%.

For the biotic additives, inducer microbes were cultivated according to the regular cultivation protocol described above. Addition of supernatant: $80 \mathrm{~mL}$ fresh medium $+10 \mathrm{~mL}$ inoculum $+10 \mathrm{~mL}$ autoclaved and centrifuged or centrifuged and sterile-filtered supernatant of inducer microbe; Addition of cell pellet: $90 \mathrm{~mL}$ fresh medium $+10 \mathrm{~mL}$ inoculum $+200-500 \mu \mathrm{L}$ autoclaved cell pellet resuspended in fresh medium.

Cell removal After termination of the cultivation, cells were removed from the broth by centrifugation in a Sorvall centrifuge by centrifuging for 20 minutes at $5000 \mathrm{rpm}$ and $4{ }^{\circ} \mathrm{C}$. Subsequently, the supernatant was filtered through folded cellulose filters with a pore size between 8 and $12 \mu \mathrm{m}$ (Sartorius Folded Filters, Grade: $3 \mathrm{hw}$, Dia: $185 \mathrm{~mm}, 65 \mathrm{~g} \cdot \mathrm{m}^{3}$, Sartorius, Göttingen). The cell pellet was collected in the respective folded filters, dried at $70^{\circ} \mathrm{C}$ for $24 \mathrm{~h}$ and weighed after drying.

Extraction of fermentation broth The cell-free broth was extracted three times with the equivalent volume of ethyl acetate. The solvent was then evaporated to dryness to gain a dry extract, which was further prepared for LC/MS measurement.

Sample preparation $2 \mathrm{~mL}$ of MS-pure methanol were added to the dry extracts, which were dissolved in the solvent via ultrasonic bath. The concentrated extract was then filtered through $0.45 \mu \mathrm{m}$ nylon filters (Chromafil ${ }^{\circledR}$ Xtra PA-45/13, Macherey\&Nagel, Düren) directly into glass vials.

LC-MS(MS) measurement The utilized LC-MS system comprised an Agilent Technologies 1260 Infinity HPLC system (Agilent Technologies, Santa Clara, USA) and a Bruker Compact ESI-QTOFMS System (Bruker, Billerica, USA). The injected sample volume was $2 \mu \mathrm{L}$. The solvents used were ACN (solvent $\mathrm{A}$ ) and $\mathrm{H}_{2} \mathrm{O}$ with $0.1 \%$ formic acid (solvent $\mathrm{B}$ ) with a flow of $0.4 \mathrm{~mL} / \mathrm{min}$. The following HPLC method was employed: 0-10 min 5\%-98\% solvent A, 10-15 min 98\% solvent A isocratic, 15-17 min $98 \%-5 \%$ solvent $\mathrm{A}, 17-20 \mathrm{~min} 5 \%$ solvent $\mathrm{A}$ at a temperature of $40^{\circ} \mathrm{C}$ with a $\mathrm{C} 18$-column (100x2.6mm NucleoShell RP18, Macherey\&Nagel, Düren). A DAD detector was used with a setting from $205 \mathrm{~nm}$ to $400 \mathrm{~nm}$. The mass spectrometer comprised an ESI ion source and a TOF analyzer and was used in positive mode. The ESI ion source was used with a nebulizing gas pressure of 4 bar, a drying gas flow of $12 \mathrm{~L} / \mathrm{min}$, a drying temperature of $220^{\circ} \mathrm{C}$ and a capillary voltage of $4500 \mathrm{~V}$. A mass-to-charge-ratio (m/z) range from 100 to 850 was measured. For some samples, Auto-MS-MS experiments were conducted. For some specific mass features, multiple reaction monitoring (MRM) measurements were used. The HPLC method was kept the same.

Evaluation of LC-MS data For the evaluation of LC-MS data, the Bruker software Compass Data Analysis 1.7 was used. The obtained data were evaluated regarding two aspects: the number of new 
mass features due to a change in cultivation conditions and the specific search for compounds whose genes were predicted by antiSMASH. In this study, a mass feature is defined as a pair of a molecular weight $\mathrm{M}_{\mathrm{w}}$ and the corresponding retention time tr. The tool MZmine 2.35 [24] was used to identify new mass features by comparison of obtained samples with control group samples and medium background as well as background of additives where necessary, e.g. supernatants. The steps and settings for raw data preparation and peak list processing, can be found in the supplementary material (Tables S9 and S10). Additionally, automated and manual refining of the obtained results was necessary to eliminate false positive hits and new mass features were compared to a list of known contaminants in mass spectrometry [25]. New mass features were numbered and named according to the producing strain $(\mathrm{BaXY}=B$. amyloliquefaciens, $\mathrm{CcXY}=\mathrm{C}$. coralloides, $\mathrm{PfXY}=P$. fallax, $\mathrm{Rj} X Y=R$. jostii, $\mathrm{SgXY}=$ S. griseochromogenes). Predicted compounds were determined by using the Extracted Ion Chromatogram (EIC) function of the Compass Data Analysis software for $\mathrm{H}^{+-}, \mathrm{NH}_{4}^{+-}, \mathrm{Na}^{+-}$and $\mathrm{K}^{+-}$ adduct identification with an allowed deviation of $0.01 \mathrm{Da}$.

Utilized databases For the literature survey, Google Scholar was used with the keywords "Bacteria OSMAC". Patents and citations were excluded. For the comparison of fragmentation patterns generated through LC/MS-MS experiments to database entries, GNPS library search was used in default mode, i.e. precursor ion mass tolerance $=2.0 \mathrm{Da}$, fragment ion mass tolerance $=0.5$ $\mathrm{Da}$, minimum number of matched peaks $=6$, score threshold $=0.7$ [26]. For the comparison of the experimental data to in silico fragmentation patterns of candidate molecules, MetFrag was used in default mode (allowed deviation from neutral mass: 5 ppm or 0.001 mzabs, mode: $[\mathrm{M}+\mathrm{H}]^{+}$, tree depth $=2$ ) with PubChem as database.

\section{Results}

Genome-mining for strain selection

42 Strains were selected based on risk group, availability and genome sequencing level as well as literature and database research. Relatively unknown representatives of phyla known for being biosynthetically interesting were chosen for the genome analysis via antiSMASH in addition to known secondary metabolite producers (NCBI accession numbers are given in Table S1). The genome analysis showed that the percentage of the bacterial genome encoding putative secondary metabolite gene clusters varies greatly among and within different phyla. On average, the selected strains dedicate $11.9 \% \pm 5.3 \%$ of their genome to secondary metabolite production, but some strains in each regarded phylum commit higher shares between 15 and over $20 \%$ of their genomes to secondary metabolite production. (Error! Reference source not found.Error! Reference source not found.) 
25

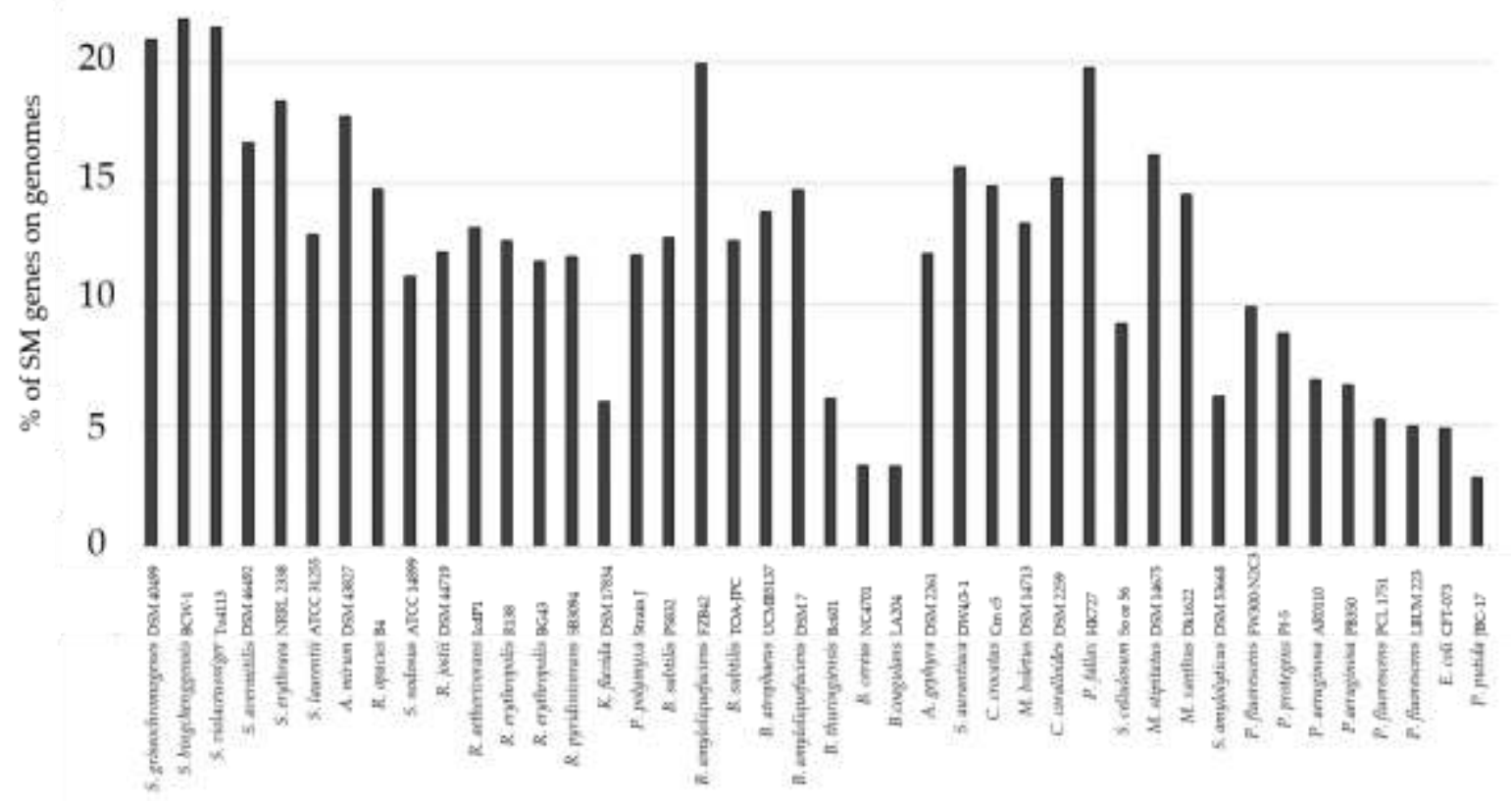

Figure 2. Percentage of secondary metabolism (SM) genes of the whole genome of selected bacteria according to antiSMASH analyses [8].

The following five strains dedicate high percentages of their genome to secondary metabolite biosynthesis, possess many BGCs for polyketides and non-ribosomal peptides as well as orphan BGCs and were, therefore, chosen for further investigation: the actinomycetes Streptomyces griseochromogenes DSM40499 (20.92\%) and Rhodococcus jostii DSM44719 (12.17\%), the proteobacteria Corallococcus coralloides DSM2259 (15.23\%) and Pyxidicoccus fallax HKI727 (19.08\%) and Bacillus amyloliquefaciens DSM7 (14.72\%) as a representative of the firmicutes. Figure 3 shows the relationship between the five selected strains. The total number of BGCs present on the genomes of selected strains varies between 11 and 49 . (

Table 1, Table S11, Table S12, Figures S2-S6,) Furthermore, only few of the predicted compounds have been reported in literature in connection with these strains so far (

Table 1), which makes the selected strains especially interesting for this study.

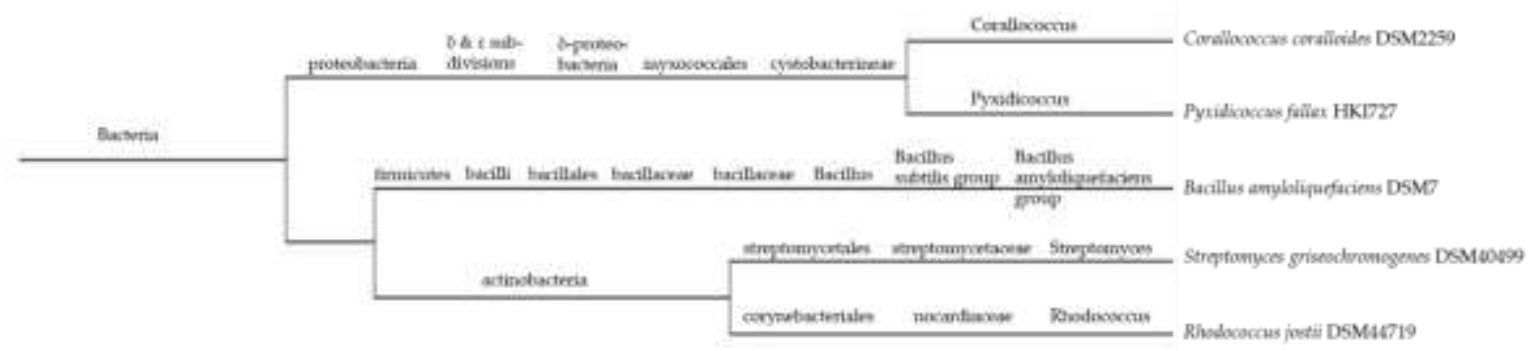

Figure 3. Phylogenetic tree of selected bacteria. Created with iTOL and adapted [27]. 
Table 1. Biosynthetic potential of selected strains in terms of \% BGCs of the whole genome and \# of BGCs and known compounds of related strains

\begin{tabular}{ccccc}
\hline Strain & $\begin{array}{c}\text { Genome } \\
\text { size [Mbp] }\end{array}$ & $\begin{array}{c}\text { \% } \\
\text { BGCs }\end{array}$ & $\begin{array}{c}\text { \# of } \\
\text { BGCs }\end{array}$ & Known compounds \\
\hline $\begin{array}{c}\text { B. amyloliquefaciens } \\
\text { DSM7 }\end{array}$ & 3.98 & 14.7 & 11 & $\begin{array}{c}\text { surfactin [28], bacillaene [29], fengycin [28], } \\
\text { bacillibactin [30] from B. amyloliquefaciens } \\
\text { FZB42 }\end{array}$ \\
\hline $\begin{array}{c}\text { C. coralloides } \\
\text { DSM2259 }\end{array}$ & 10.08 & 15.2 & 34 & - \\
\hline P. fallax HKI727 & 10.77 & 19.1 & 33 & myxochelin [31] from P. fallax HKI727 \\
\hline R. jostii DSM44719 & 7.89 & 12.2 & 18 & rhodochelin [32] from R. jostii RHA1 \\
\hline $\begin{array}{c}\text { S. griseochromogenes } \\
\text { DSM40499 }\end{array}$ & 10.76 & 20.9 & 49 & $\begin{array}{c}\text { blasticidin [33] from S. griseochromogenes (not } \\
\text { further specified) heterolously expressed in } S . \\
\text { lividans }\end{array}$ \\
\hline
\end{tabular}

Are the antiSMASH-predicted compounds produced under the tested conditions?

Overall, seven of the predicted BGCs were detected in the generated extracts. The production of five compounds was successfully activated by the variation of culture conditions, i.e. they were not detected in control group samples, while bacillaene (Figure S7) and desferrioxamine B (Figure S8) were also detected in control group samples. (Table 2, Table S13).

Table 2. Overview of number of searchable compounds, i.e. predicted non-orphan BGCs, and detected compounds in the selected bacterial strains. Compounds in bold print were activated, i.e. they were not detected in the control group.

\begin{tabular}{|c|c|c|c|c|c|c|}
\hline \multirow[b]{2}{*}{ Strain } & \multirow[b]{2}{*}{$\begin{array}{c}\# \text { of BGCs } \\
\text { according to } \\
\text { antiSMASH }\end{array}$} & \multirow{2}{*}{$\begin{array}{c}\text { \# of } \\
\text { search- } \\
\text { able com- } \\
\text { pounds } \\
\end{array}$} & \multirow{2}{*}{$\begin{array}{c}\text { Detected } \\
\text { compounds } \\
\text { (\% sequence } \\
\text { similarity) }\end{array}$} & \multicolumn{3}{|c|}{ Means of identification } \\
\hline & & & & $\begin{array}{l}\text { mass- } \\
\text { based }\end{array}$ & $\begin{array}{c}\text { MS2- } \\
\text { fragmentation }\end{array}$ & $\begin{array}{l}\text { reference } \\
\text { compound }\end{array}$ \\
\hline \multirow{3}{*}{$\begin{array}{c}\text { B. amylo- } \\
\text { liquefaciens } \\
\text { DSM7 }\end{array}$} & \multirow{3}{*}{11} & \multirow{3}{*}{6} & $\begin{array}{c}\text { surfactin } \\
(82 \%)\end{array}$ & & & $\checkmark$ \\
\hline & & & $\begin{array}{l}\text { bacillibactin } \\
(\mathbf{1 0 0} \%)\end{array}$ & & $\checkmark$ & \\
\hline & & & bacillaene $(100 \%)$ & $\checkmark$ & & \\
\hline \multicolumn{7}{|l|}{ C. coralloides } \\
\hline $\begin{array}{l}\text { DSM } \\
2259\end{array}$ & 34 & 12 & - & & & \\
\hline \multirow{2}{*}{$\begin{array}{l}\text { P. fallax } \\
\text { HKI727 }\end{array}$} & \multirow{2}{*}{33} & \multirow{2}{*}{22} & $\begin{array}{c}\text { myxochelin A } \\
(75 \%)\end{array}$ & & & $\checkmark$ \\
\hline & & & $\begin{array}{c}\text { nostophycin } \\
(\mathbf{1 8 \% )}\end{array}$ & & $\checkmark$ & \\
\hline $\begin{array}{c}\text { R. jostii DSM } \\
44719 \\
\end{array}$ & 18 & 13 & - & & & \\
\hline \multirow{2}{*}{$\begin{array}{c}\text { S. griseo- } \\
\text { chromogenes } \\
\text { DSM } \\
40499\end{array}$} & \multirow{2}{*}{49} & \multirow{2}{*}{38} & $\begin{array}{c}\text { desferrioxamine } \\
\text { B }(100 \%)\end{array}$ & & & $\checkmark$ \\
\hline & & & $\begin{array}{c}\text { albaflavenone } \\
(100 \%)\end{array}$ & $\checkmark$ & & \\
\hline
\end{tabular}

The production of surfactins by B. amyloliquefaciens was activated by diverse culture conditions: elevated temperature, minimal media use, $\mathrm{Fe}^{3+}$-limitation as well as the addition of solvents (Table S13, Figure S9). Bacillibactin on the other hand was only detected in $\mathrm{Fe}^{3+}$-limited GMS extracts. This seems reasonable due to its nature of being a siderophore [34]. (Figure S10) A compound with the mass of bacillaene was detected in extracts from several culture conditions including the control group: while the use of $\mathrm{Fe}^{3+}$-limited GMS medium and the addition of sterile-filtered supernatant of $P$. fallax led to an increase of that compound's peak area in the EIC, elevated temperature, the use of LB medium and the addition of solvents decreased its peak area, although it was still produced. Nostophycin from P. fallax was identified using MS2-fragmentation and subsequent comparison with 
an in silico fragmentation pattern using MetFrag and the corresponding nostophycin PubChem entry (PubChem ID: 101945102). (Figure S11, Figure S12, Table S14) It was detected in extracts grown on $\mathrm{Fe}^{3+}$-limited or $\mathrm{Mg}^{2+}$-limited GMS medium and when high concentrations, $6 \% \mathrm{v} / \mathrm{v}$, of ethanol or toluene were added, but by far the highest peak area was detected in the cultures with added toluene. Myxochelin A from P. fallax and putative albaflavenone from S. griseochromogenes were detected under diverse OSMAC conditions. (Table S13, Figure S13, Figure S14)

None of the compounds predicted for $C$. coralloides and $R$. jostii were detected in the respective extracts.

It was noted that high sequence similarity to database BGCs alone is not a good indicator for the biosynthetic potential of a strain. This could be seen in the example of the rhodochelin BGC in $R$. jostii and the bacilysin BGC in B. amyloliquefaciens. The corresponding compounds were not produced under any of the tested culture conditions, even though they allegedly possess $100 \%$ similarity to database BGCs. There are two possible explanations for this observation. Either the conditions needed for the activation of those BGCs have not been met yet, or the BGCs are dysfunctional. Considering the number of BGCs including the not yet annotated orphan BGCs predicted by antiSMASH, the selected strains possess even more potential that remains to be accessed.

The overview of producing conditions in Table S13 shows that especially the culture conditions with little prior literature coverage (Figure 1) are responsible for the activation of the detected and predicted secondary metabolites.

\section{Influence of selected culture conditions on the number and identity of new mass features}

The applied culture conditions successfully provoked new mass features from all tested strains. In order to assess the likelihood of the new mass features being secondary metabolites, they were categorized into groups based on their physicochemical properties, i.e. $\mathrm{M}_{\mathrm{w}}$ and $\mathrm{R}_{\mathrm{t}}$, and compared to published data of 56 bacterial primary and 128 bacterial secondary metabolites. (Figure S15, Figure S16) Table 3 summarizes the genome size, the number of predicted BGCs and the number of curated, detected new mass features.

Table 3. Genome size, numbers of predicted BGCs and numbers of detected new mass features per strain.

\begin{tabular}{cccccc}
\hline Strain & $\begin{array}{c}\text { B. amylo- } \\
\text { liquefaciens }\end{array}$ & $\begin{array}{c}\text { C. } \\
\text { coralloides }\end{array}$ & $\begin{array}{c}\boldsymbol{P} \text { fallax } \\
\text { falla }\end{array}$ & $\begin{array}{c}\boldsymbol{R} \text { jostii } \\
\text { jostiseo- }\end{array}$ & $\begin{array}{c}\text { S.griseon } \\
\text { chromogenes }\end{array}$ \\
\hline $\begin{array}{c}\text { Genome size [Mbp] } \\
\text { \# of predicted BGCs }\end{array}$ & 3.98 & 10.08 & 10.76 & 7.89 & 10.77 \\
\# of new mass features in & 11 & 34 & 33 & 18 & 49 \\
extracts & 127 & 35 & 143 & 138 & 147 \\
\hline
\end{tabular}

After data curation, 590 new mass features, which are likely to have originated from secondary metabolism, from the five strains were obtained through OSMAC experiments. This number greatly surpasses the number of encoded BGCs and verifies the assumed biosynthetic potential of the especially selected bacterial strains.

It is evident, that not all tested culture conditions are equally successful in eliciting new mass features and that the selected strains are susceptible to different conditions. (Error! Reference source $\mathbf{n}$ ot found.4-7)

Among the minimal media and limitation experiments (Figure 4), $\mathrm{PO}_{4}^{3-}-$ limitation on M9 medium ( $1 \%$ of phosphate salts of original $\mathrm{M} 9$ medium recipe used) as well as $\mathrm{Fe}^{3+-}$ and $\mathrm{Mg}^{2+-}$ limitations on GMS medium (no iron or magnesium salts used) resulted in new mass features across all tested strains. 


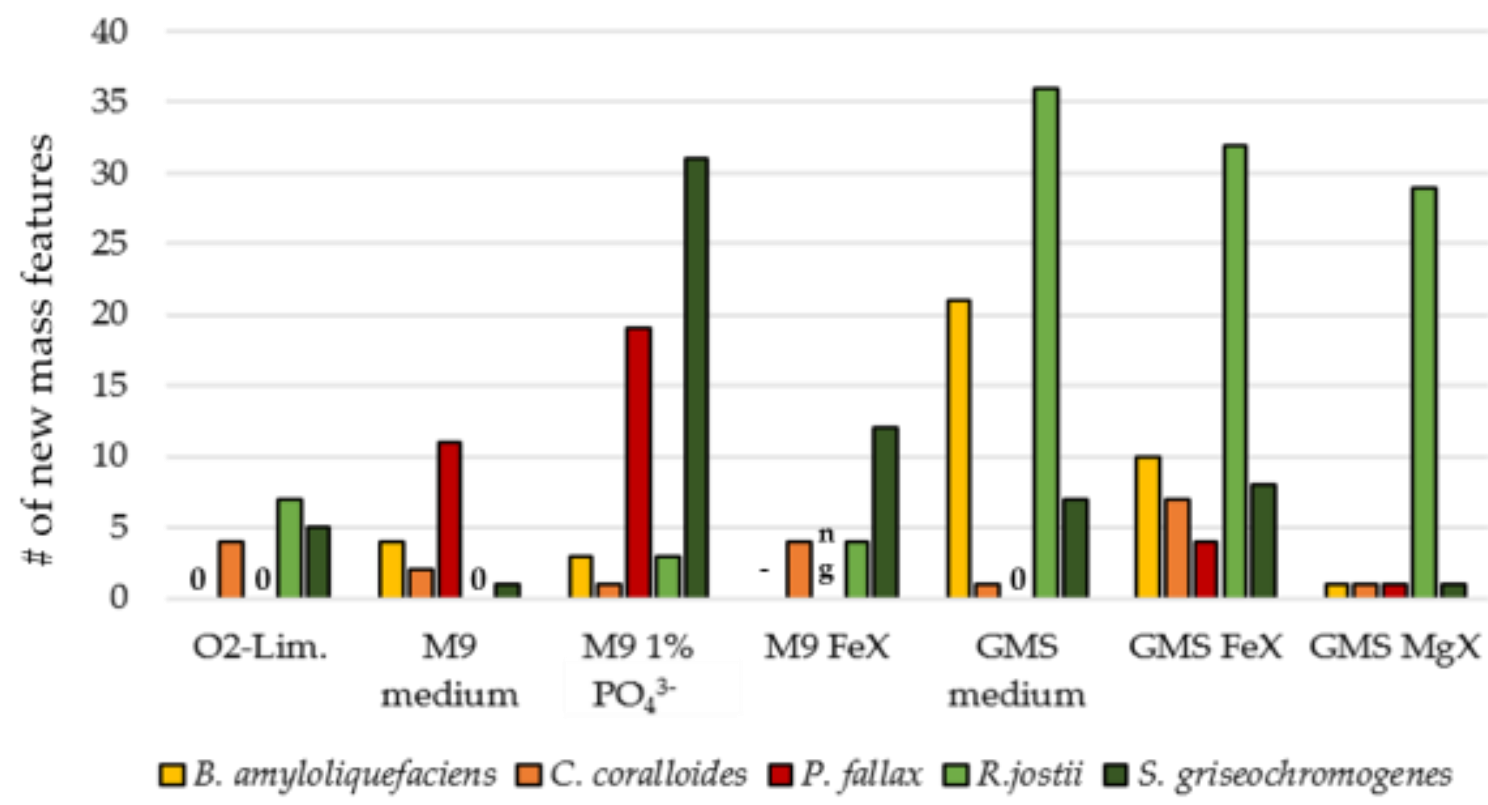

Figure 4. Number of new mass features in extracts of minimal media and limitations experiments. $=$ not tested, $0=$ no new mass features, $\mathrm{ng}=$ not grown.

This result confirms the common assumption that high $\mathrm{PO}_{4}^{3-}$-concentrations act as repressors of secondary metabolism and that a limitation of the $\mathrm{PO}_{4}^{3-}$-concentration can elicit secondary metabolism [14,35]. S. griseochromogenes and P. fallax are more susceptible to the $\mathrm{PO}_{4}{ }^{3-}$-limitation than the other tested strains, as their high numbers of new mass features in the extracts show. $R$. jostii reacted strongly to GMS medium and its limitations with up to 36 new mass features.

In the screening, we differentiated between minimal media experiments, M9 and GMS medium, and their limitations, e.g. M9 FeX and GMS FeX, so that the influence of the limitation could be seen. Therefore, new mass features from M9 or GMS medium cultures were not considered in the FeX cultures, because they obviously originated from the minimal medium and not from the $\mathrm{Fe}^{3+}$ limitation. During the evaluation it was noted that the number of new mass features varied between M9 FeX and GMS FeX cultures, e.g. four new peaks in $\mathrm{Fe}^{3+}$-limited $R$. jostii cultures on M9 medium versus 32 new peaks in $\mathrm{Fe}^{3+}$-limitation on GMS medium, and their identities were completely different. (Error! Reference source not found.) This observation shows, that the result of an $\mathrm{Fe}^{3+}-$ limitation is also dependent on the medium it is based on.

Table 5. Comparison of new mass features produced by C. coralloides and S. griseochromogenes on M9 FeX medium and GMS FeX medium. The mass features' properties ( $\mathrm{m} / \mathrm{z}$ and $\mathrm{tr}$ ) can be found in the supplementary material.

\begin{tabular}{ccc}
\hline Strain & New mass features on M9 FeX & New mass features on GMS FeX \\
\hline C. coralloides & Cc243, Cc244, Cc249, Cc250 & Cc22, Cc181, Cc186, Cc187, \\
& & Cc189, Cc193, Cc210 \\
S. & Sg115, Sg117, Sg119, Sg120, Sg135, Sg198, Sg199, & Sg96, Sg97, Sg101, Sg103, Sg104, \\
griseochromogenes & Sg201, Sg203, Sg206, Sg207, Sg214 & Sg106, Sg107, Sg109 \\
\hline
\end{tabular}

The use of complex media is a tried and trusted way of secondary metabolite discovery and BGC activation. (Figure 1) In this study, three different culture media per strain were tested and provoked new mass features in all cases but one. (Figure 5) 


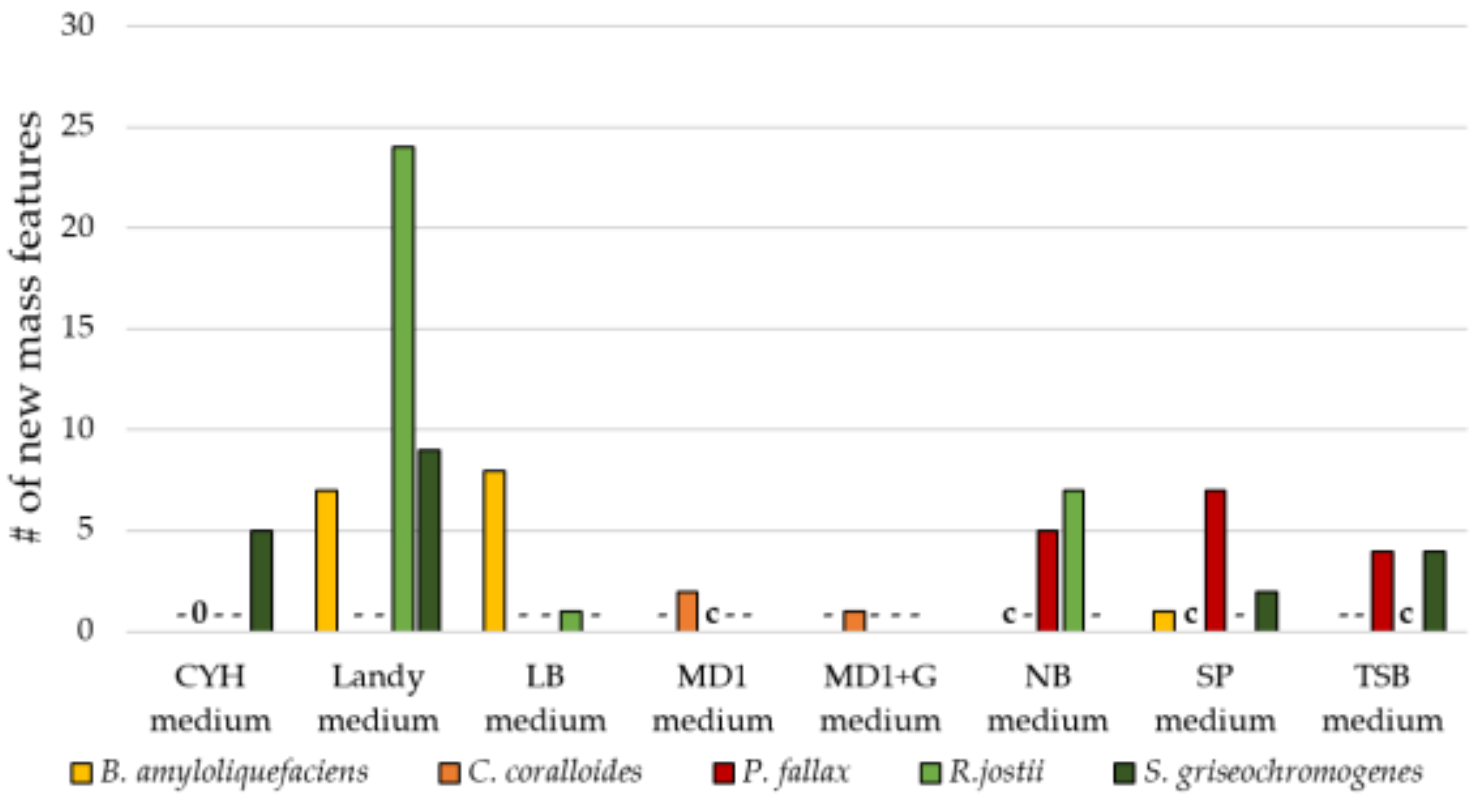

Figure 5. Number of new mass features in extracts of complex media experiments. - = not tested, $0=$ no new mass features.

The high numbers of new mass features in the Landy extracts are probably due to the difference in composition compared to the strains' control group media. While the control group media are based on complex carbon and nitrogen sources such as casitone, yeast extract or meat extract, the main components of Landy medium are glucose, glutamic acid and phenylalanine, which explains the more pronounced change in the metabolite profile.

The addition of solvents for the elicitation of secondary metabolites from bacterial strains has not been excessively reported so far. Here, this underexplored concept showed heterogeneous results across the selected strains. While $P$. fallax showed susceptibility by displaying between 5 and 41 new mass features as response to different concentrations of DMSO, ethanol and toluene, $C$. coralloides only produced 4 new mass features in total as response to all tested solvents and concentrations. (Figure 6)

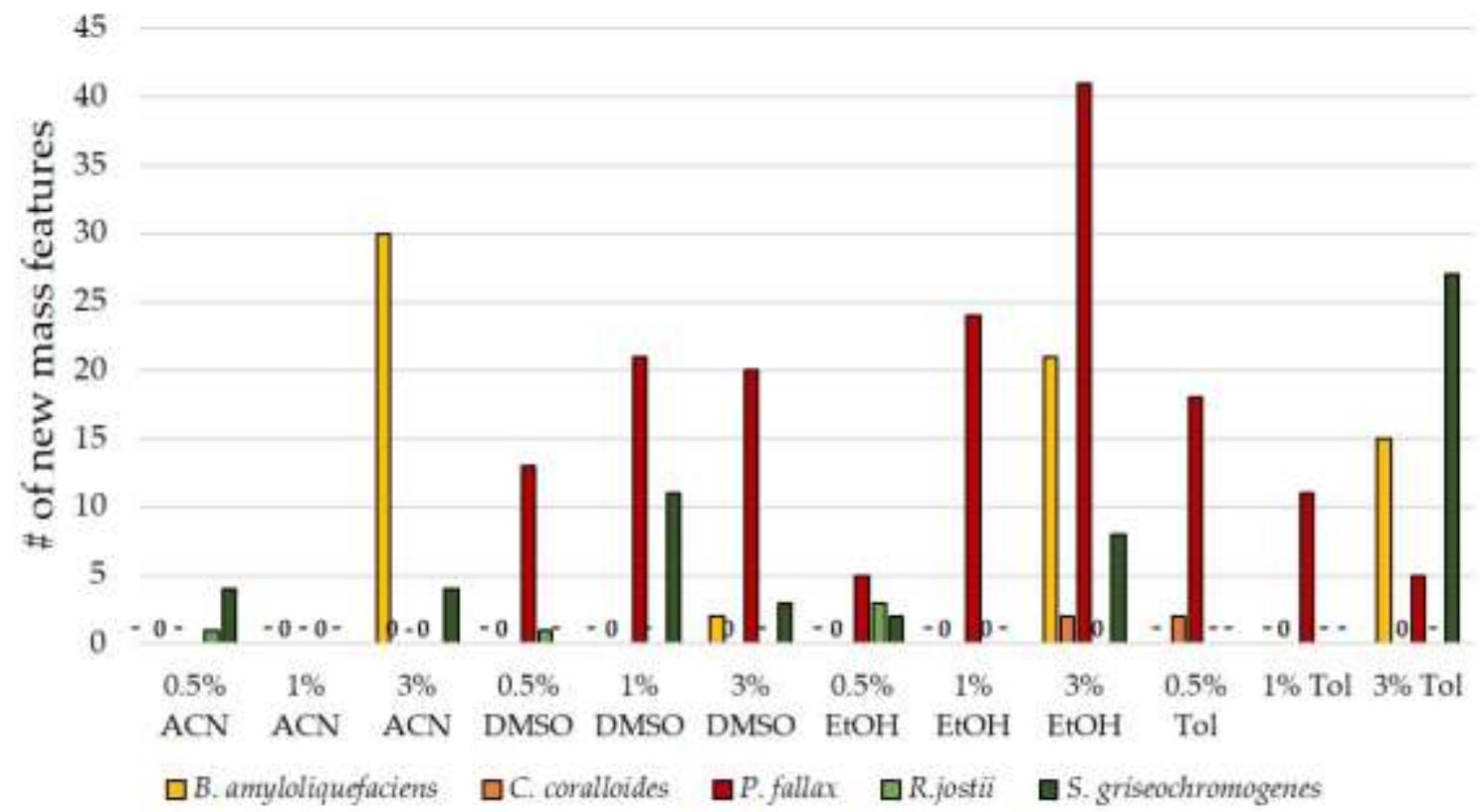

Figure 6. Number of new mass features in extracts of solvent supplementation experiments. - = not tested, $0=$ no new mass features. 
It is known that several distinct compounds are responsible for inter-species communication. Besides acyl homoserine lactones in proteobacteria signaling, peptides are known as signaling molecules in gram-positive bacteria [36]. It has been shown in manifold research papers that coculture is a productive way of exploiting the biosynthetic potential of microbes [37]. (Figure 1) The successful use of heat-killed cells for secondary metabolite elicitation has been reported previously in one research paper, which also mentions some studies from the 1990s which have dealt with the use of added supernatants [38]. Still, the use of such biotic additives has not been widely exploited (Figure 1) although it presents the possibility to mimic the presence of signal molecules and surface proteins to provoke a metabolic response in microorganisms.

The results show that new mass features were generated in both experimental setups with supernatants (sterile-filtered and autoclaved) in all tested strains. (Figure 7) Interestingly, the addition of sterile-filtered supernatant did not generally lead to higher number of new mass features as one could expect due to the pretreatment of autoclaving. This indicates that some signaling molecules must have been sufficiently temperature-stable during the autoclaving process.

In the case of $S$. griseochromogenes cultures, two new mass features were detected when supplemented with sterile-filtered supernatant from B. amyloliquefaciens and when supplemented with its autoclaved supernatant. Interestingly, the new mass features are not the same, but four different mass features were detected: Sg255 (Peak \#255 originating from a S. griseochromogenes culture) and Sg345 from sterile-filtered supernatant, Sg358 and Sg359 from autoclaved supernatant. $\mathrm{Sg} 255$ is also produced when cultures are supplemented with $0.5 \%$ toluene or sterile-filtered supernatant of $R$. jostii and Sg345 is also produced when autoclaved cell pellet of $R$. jostii is added. Sg358 and Sg359 are condition-specific and are only produced when autoclaved supernatant from $B$. amyloliquefaciens is added to $S$. griseochromogenes cultures.

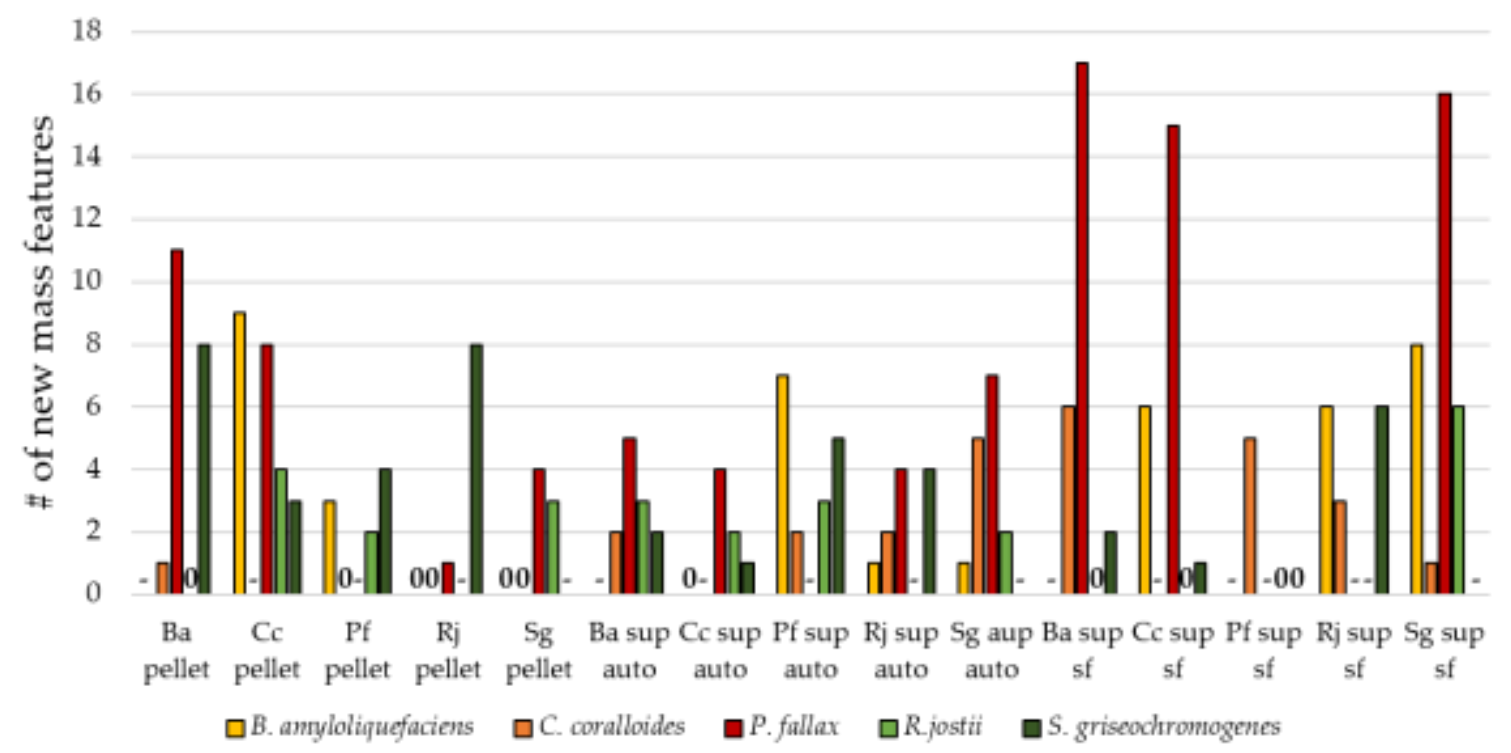

Figure 7. Number of new mass features in extracts of biotic additive experiments. $-=$ not tested, $0=$ no new mass features, $\mathrm{Ba}=B$. amyloliquefaciens, $\mathrm{Cc}=C$. coralloides, $\mathrm{Pf}=P$. fallax, $\mathrm{Rj}=R$.jostii, $\mathrm{Sg}=S$. griseochromogenes.

It cannot be concluded, that one of the supernatant types is more effective for the generation of new mass features than the other. P. fallax is the only strain with a clear benefit of the sterile-filtered supernatant: the number of new mass features is higher in all cases of added supernatants.

The addition of autoclaved cell pellet also worked well for the generation of new mass features in $P$. fallax, $R$. jostii and $S$. griseochromogenes cultures, but $C$. coralloides only reacted to the addition of B. amyloliquefaciens pellet and B. amyloliquefaciens only reacted to the myxobacteria C. coralloides and 
P. fallax. Here, we might have observed some connection to the relationship between the known predatory myxobacteria and their prey, which often constitutes Bacillus strains [39].

In contrast to the addition of organic solvents, the addition of the three types of biotic additives reliably generated new mass features in the selected strains.

Apart from C. coralloides cultures supplemented with organic solvents, the selected strains generated many new mass features under the tested culture conditions. Although only 11 BGCs were predicted by antiSMASH for B. amyloliquefaciens beforehand, a total number of 127 new mass features was discovered in its extracts (Table 3Error! Reference source not found.). $R$. jostii was expected to be a moderate producer of new mass features according to the prediction of 18 compounds, but it produced 138 new mass features. The most promising candidate was S. griseochromogenes with 49 predicted BGCs. This strain produced the most new mass features, i.e. 147. The results show that a high number of predicted BGCs does not correspond to high biosynthetic potential in terms of a high number of new mass features, as evident when comparing e.g. B. amyloliquefaciens and C. coralloides (Table 3). Overall, the selected strains produced many new mass features under the tested cultivation conditions and the selection of biosynthetically promising strains was therefore successful. Additionally, it could be shown that the culture conditions with little prior literature coverage (Figure 1), i.e. addition of solvents, biotic additives, limitations, also provoked many new mass features.

\section{Suitability of tested culture conditions for the generation of new mass features}

As expected and seen in Figure 4-7, the selected bacterial strains reacted differently to the same culture conditions. While P. fallax, for example, did not generate any new mass features on GMS medium and $C$. coralloides only managed to produce one new mass feature, B. amyloliquefaciens generated 21 new mass features on the very same medium. Overall, it was observed that all tested culture conditions are pleiotropic as they generated more than one new mass feature in at least one bacterial strain [40].

The authors agree with Bode's statement that no "common rules for all microorganisms" can be developed from OSMAC screenings and that "everything is allowed to find new natural products" as long as no complete understanding of secondary metabolism and its regulation is achieved [14]. Still, based on our findings, we want to postulate that some culture conditions can be regarded as more promising than others and constitute good starting points for future screenings. Therefore, we evaluated the selected culture conditions regarding their capability to provoke new mass features from different strains. This capability is termed hit rate and defined as the number of strains generating new mass features in response to one culture condition over the number of tested strains. The results show that $36 \%$ of the tested culture conditions led to the detection of new mass features in all strains they were tested with. This corresponds to a hit rate of 1 . (Figure 8)

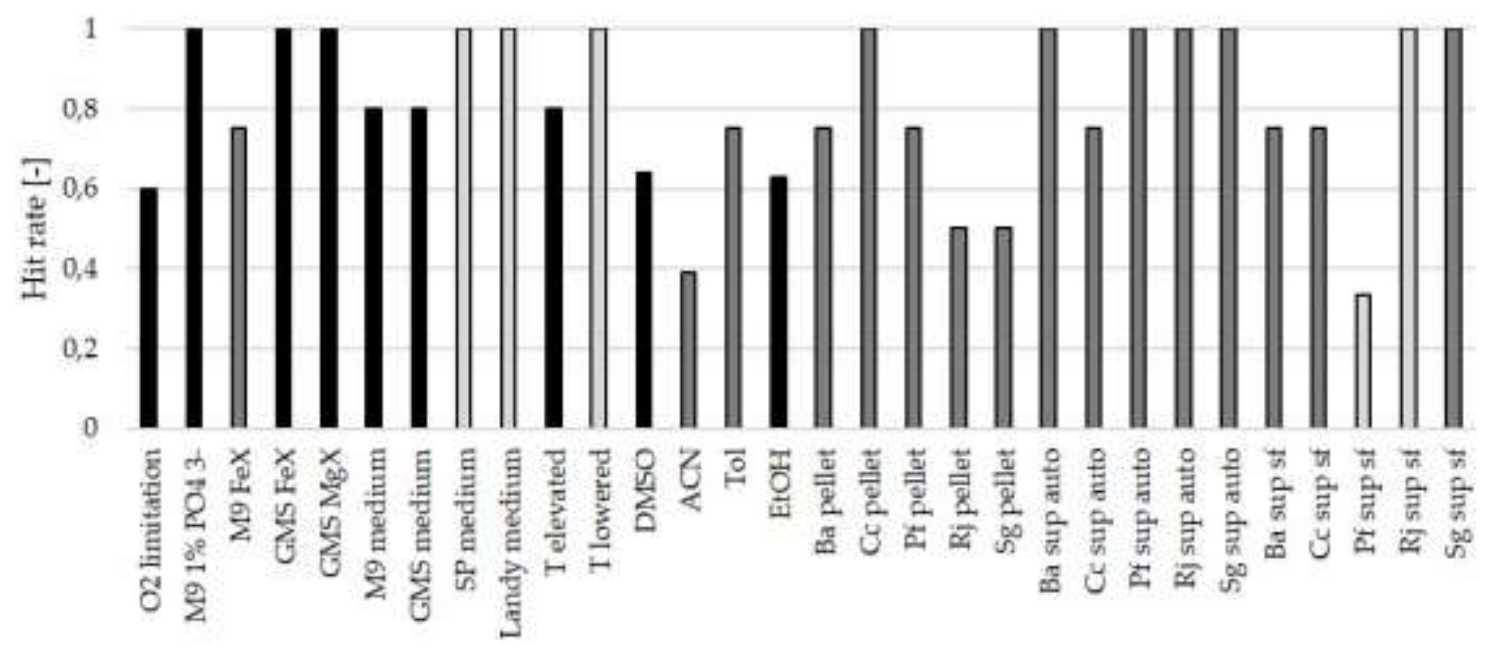

Figure 8. Hit rates for all tested culture conditions. Black bars: sample size $\mathrm{n}=5$ strains, dark grey bars: $\mathrm{n}=4$, light grey bars: $n=3$. 
The addition of several biotic additives (cell pellet of $C$. coralloides, autoclaved supernatants of all strains but $C$. coralloides) and some limitation experiments (phosphate limitation on M9 medium and $\mathrm{Fe}^{3+}$-and $\mathrm{Mg}^{2+}$-limitation on GMS medium) have reliably provoked new mass features from all strains they were tested with and can be recommended as starting points for future screenings with other bacteria. It should be kept in mind, that the results of the limitation experiments were dependent on the medium as well, see M9 FeX versus GMS FeX, and therefore the exact condition of the limitation experiments, i.e. $\mathrm{Fe}^{3+}-$ limitation on GMS medium and not $\mathrm{Fe}^{3+}$-limitation alone, is recommended.

Our analysis also shows that in most tested strains over $70 \%$ of the generated new mass features were only produced under exactly one of the tested culture conditions (condition-specific). Between 7 and $19 \%$ of the new mass features were generated under two culture conditions and even less under three or more culture conditions. (Figure 9)

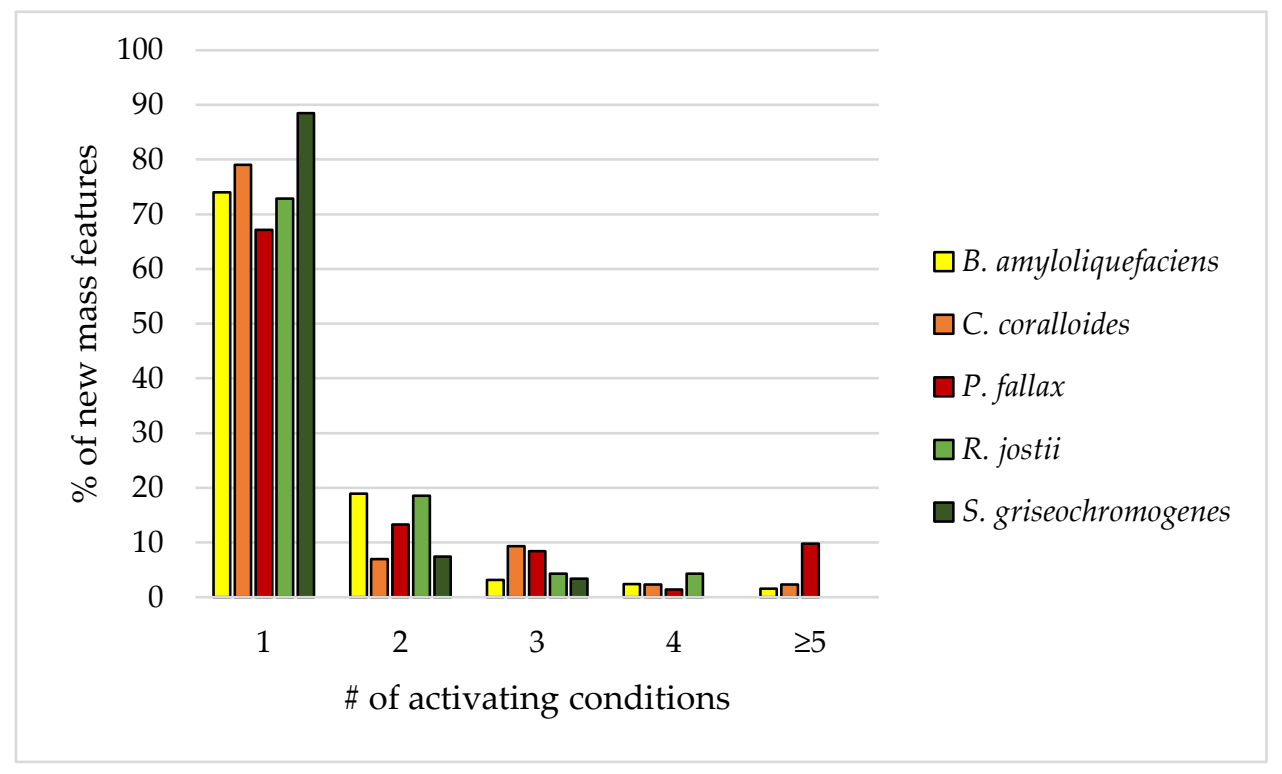

Figure 9. Percentage of new mass features, which are activated by a certain number of culture conditions.

Due to the observed condition-specificity of the new mass features, it can be postulated that rather a broad screening of many culture conditions with a high hit rate applied to carefully selected and promising bacterial strains is a recommendable way of approaching the search for novel bacterial secondary metabolites.

Are there compounds among the new peaks which are so far unreported for the strains?

The analysis of LC/MS data resulted in detailed lists of new mass features for each investigated strain containing $M_{w}$, tRS and cultivation conditions (Tables S15-S19). The 590 new mass features were evaluated regarding their intensity and peak area with heat maps for prioritization. (Tables S17-S19) The top $5 \%$ regarding peak area were subjected to LC-MS2 measurements to obtain their compoundspecific fragmentation patterns. Identified mass features, their corresponding compounds and means of identification are listed in Table 4. The corresponding producing conditions are listed in Table S20. 
Table 4. Results of fragmentation pattern analysis of selected new mass features. Evidence is given in the supplementary material.

\begin{tabular}{|c|c|c|c|c|c|c|}
\hline \multirow{2}{*}{ ID } & \multirow{2}{*}{$\mathbf{m} / \mathbf{z}$} & \multirow{2}{*}{$\begin{array}{c}t_{R} \\
{[\mathrm{~min}]}\end{array}$} & \multirow{2}{*}{ Proposed compound } & \multicolumn{3}{|c|}{ Means of identification } \\
\hline & & & & GNPS & MetFrag & Reference compound \\
\hline Ba3 & 261.12 & 4.5 & cyclo(Tyr-Pro) & $\checkmark$ & & $\checkmark$ \\
\hline Ba8 & 1057.57 & 7.2 & iturin A-4 & $\checkmark$ & $\checkmark$ & \\
\hline Ba9 & 1071.58 & 7.6 & iturin A-6 & \multicolumn{3}{|c|}{ mass-based } \\
\hline Ba10 & 1085.6 & 7.9 & iturin A-8 & \multicolumn{3}{|c|}{$\checkmark$} \\
\hline Ba58 & 883.26 & 6.1 & bacillibactin & \multicolumn{3}{|c|}{$\begin{array}{l}\text { comparison to fragmentation pattern from } \\
\text { literature } \\
\end{array}$} \\
\hline Cc42 & 601.36 & 4.7 & nocardamin & $\checkmark$ & & $\checkmark$ \\
\hline Pf336 & 405.16 & 5.4 & myxochelin A & & & $\checkmark$ \\
\hline Sg117 & 587.35 & 4.5 & $\begin{array}{c}\text { desmethyl enyl } \\
\text { nocardamin }\end{array}$ & $\checkmark$ & & \\
\hline Sg130 & 585.36 & 4.9 & desferrioxamine $\mathrm{B}+\mathrm{Al}$ & $\checkmark$ & & \\
\hline
\end{tabular}

Since those compounds are represented by new mass features, they were not produced in the control group and thus activated by the variation of culture conditions. The production of bacillibactin (Ba58), myxochelin A (Pf336) and desferrioxamine B (Sg117 derivative, Sg130 Alcomplex) was already described above, as their BGCs were predicted by antiSMASH.

Ba3, which has been identified as cyclo-(Tyr-Pro) (Figure S20), is a cyclic dipeptide with antimicrobial activity against gram negative strains and has so far not been reported in literature for strain B. amyloliquefaciens DSM7, although strains from the B. subtilis group, are generally regarded as producers of cyclic peptides [41,42]. It is produced under various culture conditions: elevated temperature, $\mathrm{Mg}^{2+}-$ limitation and two different complex media.

The mass features named Ba8, Ba9 and Ba10 have been putatively matched with iturins A-4, A6 and A-8 (Figures S21-S24). Interestingly, no iturin BGC was detected by antiSMASH, although it is a common antifungal secondary metabolite of B. amyloliquefaciens strains [43]. In fact, a detailed analysis of the B. amyloliquefaciens DSM7 genome independent of antiSMASH conducted in 2011, revealed an iturin BGC and a follow-up study is the only literature report of iturin production by this exact strain $[44,45]$. In the study by Borriss et al. iturin A was detected in DSM7 cultures grown in Landy medium [45], while we detected the compound in LB cultures. Since our antiSMASH genome analysis revealed an orphan NRPS BGC on the genome of B. amyloliquefaciens DSM7, it is possible that this is the missing iturin A BGC. This assumption remains to be investigated.

Cc42 has been identified as nocardamin, a cyclic desferrioxamine B derivative, and as such a siderophore [46]. (Figure S25) This compound has not been reported in literature for this strain so far. As a matter of fact, nocardamin has only been detected in actinomycete strains, mostly in Streptomyces cultures, and has, to our best knowledge, never been reported for any proteobacterial strain before. Our antiSMASH analysis has not revealed the nocardamin BGC, but the C. coralloides DSM2259 genome harbors many orphan BGCs, so that the nocardamin BGC remains to be annotated.

Streptomycetes are known producers of desferrioxamine siderophores, which include nocardamin (=desferrioxamine E) [47]. Different desferrioxamine derivatives have been detected in our S. griseochromogenes cultures, but the corresponding BGC was already active in the control group. It has been observed that a change in culture conditions influences the distribution of derivatives (data not shown) and the BGC can also be turned off. Mass feature Sg117, desmethyl-enyl nocardamin, was only detected in the iron-deprived M9 culture (M9 FeX) and the complex of desferrioxamine B with aluminum was only detected in cultures supplemented with DMSO. (Figures S26, S27) 


\section{Conclusions}

The biosynthetically promising and fully sequenced bacterial strains B. amyloliquefaciens DSM7, C. coralloides DSM2259, P. fallax HKI727, R. jostii DSM77419 and S. griseochromogenes DSM40499 were selected for OSMAC experiments based on genome mining, availability and literature research in order to elucidate their biosynthetic potential. Among all strains, five BGCs could be activated using OSMAC conditions: surfactin variants and bacillibactin from B. amyloliquefaciens extracts, myxochelin $A$ and putatively nostophycin from $P$. fallax extracts and putatively albaflavenone from $S$. griseochromogenes extracts. Additionally, bacillaene and desferrioxamine B were detected in cultures of B. amyloliquefaciens and S. griseochromogenes respectively. Since these two compounds were already present in control group samples their BGCs were not activated. Still, some BGCs remained silent under the tested culture conditions, some even despite $100 \%$ sequence similarity, which indicates that high sequence similarity alone is not a good indicator for the biosynthetic potential of strains, as could be seen in the examples of B. amyloliquefaciens and R. jostii.

The high number of provoked new mass features, e.g. 147 new peaks in S. griseochromogenes DSM40499 extracts, validates the expected high biosynthetic potential of the selected strains. $B$. amyloliquefaciens DSM7 and R. jostii DSM77419 surpassed the expectations with their high numbers of new peaks, 127 for B. amyloliquefaciens DSM7 and 138 for $R$. jostii DSM44719, in comparison to the number of predicted BGCs (Table 3). Additionally, the genomes of the investigated strains still possess high numbers of orphan BGCs. These findings indicate that the biosynthetic potential of the investigated strains has not been fully elucidated so far and holds more to discover. Among the new mass features some have been identified as interesting compounds, which had not been predicted beforehand: the bioactive cyclic dipeptide cyclo(Tyr-Pro) and the known Bacillus-lipopeptide iturin from B. amyloliquefaciens DSM7 and the siderophore nocardamin from C. coralloides DSM2259 which are all unreported for these strains. It remains to be investigated if any of the identified new mass features are products originating from orphan BGCs.

The culture conditions with little literature coverage have successfully contributed to the activation of predicted BGCs as well as to the generation of new mass features.

An analysis of the tested culture conditions and the new mass features revealed that some culture conditions provoke the production of new mass features from the tested bacterial strains more reliably than others. Another finding was the condition-specificity of over $70 \%$ of generated new mass features. From these observations we draw the conclusion that firstly, $\mathrm{PO}_{4}{ }^{3-}-\mathrm{limited} \mathrm{M} 9$ medium, $\mathrm{Fe}^{3+}-$ limited GMS medium and several biotic additives are promising starting points for future screenings and secondly, that a broad screening with many tested culture conditions and a small number of carefully selected strains is a promising approach for the search for novel bacterial secondary metabolites due to the condition-specificity of mass features.

Supplementary Materials: The following are available online at www.mdpi.com/xxx/s1, Figure S1: title, Table S1: title, Video S1: title.

Author Contributions: Conceptualization, J.S. and S.L.; methodology, J.S.; software, J.S.; validation, J.S.; formal analysis, J.S.; investigation, J.S.; resources, S.L.; data curation, J.S.; writing-original draft preparation, J.S.; writing-review and editing, S.L.; visualization, J.S.; supervision, S.L.; project administration, S.L.; funding acquisition, S.L. All authors have read and agreed to the published version of the manuscript.

Funding: This research received no external funding.

Acknowledgments: The authors would like to acknowledge the scientific advice and help with analytics provided by C. Zammarelli as well as the scientific exchange with Prof. M. Nett, Dipl.-Pharm. A. Sester and Dr. K. Rosenthal, which enriched this study. We acknowledge financial support by Deutsche Forschungsgemeinschaft and Technische Universität Dortmund/TU Dortmund University within the funding programme Open Access Publishing.

Conflicts of Interest: The authors declare no conflict of interest.

\section{References}


[1] McMurry, J. Secondary Metabolites: An Introduction to Natural Products Chemistry. In Organic Chemistry with Biological Applications,3rd ed.; Cengage Learning: Stamford, CT, USA, 2015; pp. 1016-1046.

[2] Luckner, M. What is Secondary Metabolism? In Secondary Metabolism in Microorganisms, Plants, and Animals, 3rd ed.; Springer Verlag: Berlin, 1990; pp.15-23.

[3] Newman, D.J.; Cragg, G.M. Natural Products as Sources of New Drugs over the Nearly Four Decades from 01/1981 to 09/2019. J.Nat.Prod. 2020, 83, 770-803.

[4] Wu, C.; Zacchetti, B.; Ram, A. F. J.; Van Wezel, G. P.; Claessen, D.; Choi, Y. H. Expanding the chemical space for natural products by Aspergillus-Streptomyces co-cultivation and biotransformation. Sci. Rep. 2015, 5, 1-10.

[5] Surup, F.; Viehrig, K.; Mohr, K.I.; Herrmann, J.; Jansen, R.; Müller, R. Disciformycins A and B: 12-membered macrolide glycoside antibiotics from the myxobacterium pyxidicoccus fallax active against multiresistant staphylococci. Angew. Chemie - Int. Ed. 2014, 53, 13588-13591.

[6] Rateb, M. E.; Houssen, W. E.; Harrison, W. T. A.; Deng, H.; Okoro, C. K.; Asenjo, J. A.; Andrews, B. A.; Bull, A. T.; Goodfellow, M. ; Ebel, R. ; Jaspars, M. Diverse Metabolic Profiles of a Streptomyces Strain Isolated from a Hyper-arid Environment. J. Nat. Prod. 2011, 74, 1965-1971.

[7] Jamil, B.; Hasan, F.; Hameed, A.; Ahmed, S. Isolation of bacillus subtilis MH-4 from soil and its potential of polypeptidic antibiotic production. Pak. J. Pharm. Sci. 2007, 20, 26-31.

[8] Weber, T.; Blin, K.; Duddela, S.; Krug, D.; Kim, H. U.; Bruccoleri, R.; Lee, S. Y.; Fischbach, M. A.; Müller, R.; Wohlleben, W.; Breitling, R.; Takano, E.; Medema, M. H. AntiSMASH 3.0-A comprehensive resource for the genome mining of biosynthetic gene clusters. Nucleic Acids Res. 2015, 43, W237-W243.

[9] Wilkinson, B.; Micklefield, J. Mining and engineering natural-product biosynthetic pathways. Nat. Chem. Biol. 2007, 3, 379-386.

[10] Okada, B. K.; Seyedsayamdost, M. R. Antibiotic dialogues: Induction of silent biosynthetic gene clusters by exogenous small molecules. FEMS Microbiol. Rev. 2017, 41, 19-33.

[11] Zarins-Tutt, J. S.; Barberi, T. T.; Gao, H.; Mearns-Spragg, A.; Zhang, L.; Newman, D. J.; Goss R. J. M. Prospecting for new bacterial metabolites: A glossary of approaches for inducing, activating and upregulating the biosynthesis of bacterial cryptic or silent natural products. Nat. Prod. Rep. 2016, 33, 54-72.

[12] Scherlach, K.; Hertweck, C. Triggering cryptic natural product biosynthesis in microorganisms. Org. Biomol. Chem. 2009, 7, 1753-1760.

[13] Rackham, E. J.; Grüschow, S.; Ragab, A. E.; Dickens, S.; Goss, R. J. M. Pacidamycin biosynthesis: Identification and heterologous expression of the first uridyl peptide antibiotic gene cluster. ChemBioChem 2010, 11, 1700-1709.

[14] Bode, B. H.; Bethe, B.; Höfs, R.; Zeeck, A. Big Effects from Small Changes: Possible Ways to Explore Nature's Chemical Diversity. ChemBioChem 2002, 3, 619-627.

[15] Bode, H. B.; Walker, M.; Zeeck, A. Secondary metabolites by chemical screening. 42 Cladospirones B to I from Sphaeropsidales sp. F-24'707 by variation of culture conditions. European J. Org. Chem. 2000, 31853193.

[16] van Wezel, G. P.; Wu, C.; Hankemeier, T.; Elsayed, S. S.; Machushynets, N. V. Discovery of novel glycerolated quinazolinones from Streptomyces sp. MBT27. J. Ind. Microbiol. Biotechnol. 2019, DOI 10.1007/s10295-019-02140-2.

[17] Li, W.; Ding, L.; Wang, N.; Xu, J.; Zhang, W.; Zhang, B.; He, S.; Wu, B.; Jin, H. Isolation and Characterization of Two New Metabolites from the Sponge-Derived Fungus Aspergillus sp. LS34 by OSMAC Approach. Mar. Drugs 2019, 17, 283.

[18] Chen, G.; Wang, G.; Li, X.; Waters, B.; Davies, J. Enhanced Production of Microbial Metabolites in the Presence of Dimethyl Sulfoxide. J. Antibiot. (Tokyo). 2000, 53, 1145-1153.

[19] Lindquist, S. The Heat-Shock Response. Annu. Rev. Biochem. 1986, 55, 1151-1191.

[20] Weinberg, E. D. Roles of trace metals in transcriptional control of microbial secondary metabolism. Biol. Met. 1990, 191-196.

[21] Paranagama, P. A.; Wijeratne, E. M.; Gunatilaka, A. A. Uncovering Biosynthetic Potential of PlantAssociated Fungi: Effect of Culture Conditions on Metabolite Production by Paraphaeosphaeria quadriseptata and Chaetomium chiversii(1). J Nat Prod 2007, 21, 0.

[22] Freemont, P.; Polizzi, K. M.; Goers, L. Co-culture systems and technologies: taking synthetic biology to the next level. J. R. Soc. Interface 2014, 11, DOI 10.1098/rsif.2014.0065.

[23] Moody, S. C. Microbial co-culture: harnessing intermicrobial signaling for the production of novel antimicrobials. Future Microbiol. 2014, 9, 575-578.

[24] Pluskal, T.; Castillo, S.; Villar-Briones, A.; Orešič, M. MZmine 2: Modular framework for processing, visualizing, and analyzing mass spectrometry-based molecular profile data. BMC Bioinformatics 2010, 11, DOI 10.1186/1471-2105-11-395. 
[25] Keller, B. O.; Sui, J.; Young, A. B.; Whittal, R. M. Interferences and contaminants encountered in modern mass spectrometry. Anal. Chim. Acta 2008, 627, 71-81; DOI 10.1016/j.aca.2008.04.043.

[26] Wang, M.; Carver, J. J.; Phelan, V. V.; Sanchez, L. M.; Garg, N.; Peng, Y.; Nguyen, D. D.; Watrous, J.; Kapono, C. A.; Luzzatto-Knaan, T.; Porto, C.; Bouslimani, A.; Melnik, A. V.; Meehan, M. J.; Liu, W. T.; Crüsemann, M.; Boudreau, P. D.; Esquenazi, E.; Sandoval-Calderón, M.; Kersten, R. D.; Pace, L. A.; Quinn, R. A.; Duncan, K. R.; Hsu, C. C.; Floros, D. J.; Gavilan, R. G.; Kleigrewe, K.; Northen, T.; Dutton, R. J.; Parrot, D.; Carlson, E. E.; Aigle, B.; Michelsen, C. F.; Jelsbak, L.; Sohlenkamp, C.; Pevzner, P.; Edlund, A.; McLean, J.; Piel, J.; Murphy, B. T.; Gerwick, L.; Liaw, C. C.; Yang, Y. L.; Humpf, H. U.; Maansson, M.; Keyzers, R. A.; Sims, A. C.; Johnson, A. R.; Sidebottom, A. M.; Sedio, B. E.; Klitgaard, A.; Larson, C. B.; Boya, C. A. P.; Torres-Mendoza, D.; Gonzalez, D. J.; Silva, D. B.; Marques, L. M.; Demarque, D. P.; Pociute, E.; O’Neill, E. C.; Briand, E.; Helfrich, E. J. N.; Granatosky, E. A.; Glukhov, E.; Ryffel, F.; Houson, H.; Mohimani, H.; Kharbush, J. J.; Zeng, Y.; Vorholt, J. A.; Kurita, K. L.; Charusanti, P.; McPhail, K. L.; Nielsen, K. F.; Vuong, L.; Elfeki, M.; Traxler, M. F.; Engene, N.; Koyama, N.; Vining, O. B.; Baric, R.; Silva, R. R.; Mascuch, S. J.; Tomasi, S.; Jenkins, S.; Macherla, V.; Hoffman, T.; Agarwal, V.; Williams, P. G.; Dai, J.; Neupane, R.; Gurr, J.; Rodríguez, A. M. C.; Lamsa, A.; Zhang, C.; Dorrestein, K.; Duggan, B. M.; Almaliti, J.; Allard, P. M.; Phapale, P.; Nothias, L. F.; Alexandrov, T.; Litaudon, M.; Wolfender, J. L.; Kyle, J. E.; Metz, T. O.; Peryea, T.; Nguyen, D. T.; VanLeer, D.; Shinn, P.; Jadhav, A.; Müller, R.; Waters, K. M.; Shi, W.; Liu, X.; Zhang, L.; Knight, R.; Jensen, P. R.; Palsson, B.; Pogliano, K.; Linington, R. G.; Gutiérrez, M.; Lopes, N. P.; Gerwick, W. H.; Moore, B. S.; Dorrestein, P. C.; Bandeira, N. Sharing and community curation of mass spectrometry data with Global Natural Products Social Molecular Networking. Nat. Biotechnol. 2016, 34, 828-837.

[27] Letunic, I.; Bork, P. Interactive Tree of Life (iTOL) v4: Recent updates and new developments. Nucleic Acids Res. 2019, 47, 256-259.

[28] Cawoy, H..; Mariutto, M.; Henry, G.; Fisher, C.; Vasilyeva, N.; Thonart, P.; Dommes, J.; Ongena, M. Plant Defense Stimulation by Natural Isolates of Bacillus Depends on Efficient Surfactin Production. Mol. PlantMicrobe Interact.

[29] Chen, X. H.; Scholz, R.; Borriss, M.; Junge, H.; Mögel, G.; Kunz, S.; Borriss, R. Difficidin and bacilysin produced by plant-associated Bacillus amyloliquefaciens are efficient in controlling fire blight disease. J. Biotechnol. 2009, 140, 38-44.

[30] Dunlap, C. A.; Bowman, M. J.; Schisler, D. A. Genomic analysis and secondary metabolite production in Bacillus amyloliquefaciens AS 43.3: A biocontrol antagonist of Fusarium head blight. Biol. Control 2013, 64, 166-175.

[31] Korp, J.; König, S.; Schieferdecker, S.; Dahse, H. M.; König, G. M.; Werz, O.; Nett, M. Harnessing Enzymatic Promiscuity in Myxochelin Biosynthesis for the Production of 5-Lipoxygenase Inhibitors. ChemBioChem 2015, 16, 2445-2450.

[32] Bosello, M.; Robbel, L.; Linne, U.; Xie, X.; Marahiel, M. A. Biosynthesis of the siderophore rhodochelin requires the coordinated expression of three independent gene clusters in Rhodococcus jostii RHA1. J. Am. Chem. Soc. 2011, 133, 4587-4595.

[33] Cone, M. C.; Yin, X.; Grochowski, L. L.; Parker, M. R.; Zabriskie, T. M. The blasticidin S biosynthesis gene cluster from Streptomyces griseochromogenes: Sequence analysis, organization, and initial characterization. ChemBioChem 2003, 4, 821-828.

[34] Miethke, M.; Westers, H.; Blom, E. J.; Kuipers, O. P.; Marahiel, M. A. Iron starvation triggers the stringent response and induces amino acid biosynthesis for bacillibactin production in Bacillus subtilis. J. Bacteriol. 2006, 188, 8655-8657.

[35] Masuma, R.; Tanaka, Y.; Tanaka, H.; Omura, S. Production of Nanaomycin and other Antibiotics by Phosphate-depressed Fermentation Using Phosphate-trapping Agents. J. Antibiot. (Tokyo). 1986, 39, 15571564.

[36] Tobias, N. J.; Brehm, J.; Kresovic, D.; Brameyer, S.; Bode, H. B.; Heermann, R. New Vocabulary for Bacterial Communication. ChemBioChem 2019, 1-11.

[37] Pettit, R. K. Mixed fermentation for natural product drug discovery. Appl. Microbiol. Biotechnol. 2009, 83, $19-25$.

[38] Mearns-Spragg, A.; Bregu, M.; Boyd, K.G.; Burgess, J.G. Cross-species induction and enhancement of antimicrobial activity produced by epibiotic bacteria from marine algae and invertebrates, after exposure to terrestrial bacteria. Lett. Appl. Microbiol. 1998, 142-146.

[39] Hamoen, L. W.; Venema, G.; Kuipers, O. P. Controlling competence in Bacillus subtilis: Shared use of regulators. Microbiology 2003, 149, 9-17.

[40] Rutledge, P. J.; Challis, G. L. Discovery of microbial natural products by activation of silent biosynthetic gene clusters. Nat. Rev. Microbiol. 2015, 13, 509-523.

[41] Milne, P. J.; Hunt, A. L.; Rostoll, K.; Van Der Walt, J. J.; Graz, C. J. M. The Biological Activity of Selected 
Cyclic Dipeptides. J. Pharm. Phamacol 1998, 50, 1331-1337.

[42] Pomilio, A.; Battista, M.; Vitale, A. Naturally-Occurring Cyclopeptides: Structures and Bioactivity. Curr. Org. Chem. 2006, 10, 2075-2121.

[43] Koumoutsi, A.; Henne, A.; Liesegang, H.; Hitezeroth, G.; Franke, P.; Vater, J.; Borriss, R. Structural and Functional Characterization of Gene Clusters Directing Nonribosomal Synthesis of Bioactive Cyclic Lipopeptides. J. Bacteriol. 2004, 186, 1084-1096.

[44] Rückert, C.; Blom, J.; Chen, X. H.; Reva, O.; Borriss, R. Genome sequence of B. amyloliquefaciens type strain DSM7T reveals differences to plant-associated B. amyloliquefaciens FZB42. J. Biotechnol. 2011, 155, 78-85.

[45] Borriss, R.; Chen, X. H.; Rueckert, C.; Blom, J.; Becker, A.; Baumgarth, B.; Fan, B.; Pukall, R.; Schumann, P.; Spröer, C.; Junge, H.; Vater, J.; Pühler, A.; Klenk, H. P. Relationship of Bacillus amyloliquefaciens clades associated with strains DSM 7T and FZB42T: a proposal for Bacillus amyloliquefaciens subsp. amyloliquefaciens subsp. nov. and Bacillus amyloliquefaciens subsp. plantarum subsp. nov. based on complete genome sequence comparisons. Int. J. Syst. Evol. Microbiol. 2011, 61, 1786-1801.

[46] Lee, H. S.; Hee, J. S.; Kyoung, H. J.; Tae, S. K.; Oh, K. B.; Shin, J. Cyclic peptides of the nocardamine class from a marine-derived bacterium of the genus Streptomyces. J. Nat. Prod. 2005, 68, 623-625.

[47] Yamanaka, K.; Oikawa, H.; Ogawa, H. O.; Hosono, K.; Shinmachi, F.; Takano, H.; Sakuda, S.; Beppu, T.; Ueda, K. Desferrioxamine E produced by Streptomyces griseus stimulates growth and development of Streptomyces tanashiensis. Microbiology 2005, 151, 2899-2905. 